\title{
6. Sind menschliche zerebrale Organoide moralisch schützenswert? Ein kommentierter Überblick über die aktuelle internationale Ethikdiskussion 1
}

\subsection{Einleitung: Wie Mr. Brown und Mr. Robinson die Diskussion eröffneten}

Anfang der 1960er Jahre publizierte der amerikanische Philosoph Sydney Shoemaker ein später recht viel diskutiertes Buch über personale Identität (1963). Darin entwickelte Shoemaker ein Gedankenexperiment: Stellen Sie sich vor, es wäre möglich, das Gehirn von Herrn Brown in Herrn Robinson zu verpflanzen, dessen eigenes Gehirn zuvor entfernt wurde. Wer ist dann diese Person? Herr Brown, Herr Robinson oder eine neue Person, die Shoemaker als „Mr. Brownson“ bezeichnet? Shoemaker diskutierte damit die Relevanz der materialen, verkörperlichten Grundlage von personaler Identität und stützt dabei ein wichtiges Paradigma der Bioethik, den sogenannten Neurozentrismus. Dem Neurozentrismus zufolge kommt den anatomisch-physiologischen Bedingungen des menschlichen Geistes eine große, gar entscheidende Relevanz zu. Die späteren Dekaden der Neurowissenschaften und der Neurophilosophie sind großenteils davon geprägt, die Bedeutung organischer Grundlagen des Geistes zu klären. Unumstritten hat diese Diskussion auch ethische Implikationen: Die ethischen Kontroversen um die Gleichsetzung des Hirntodes mit dem menschlichen Tod, um Experimente am menschlichen Gehirn oder über die Verpflanzung von tierischem, neuronalen Gewebe in den Menschen zeichnen sich dadurch aus, dass moralische $\mathrm{Zu}$ schreibungen aus den Eigenschaften der jeweiligen Neurosubstanz abgeleitet werden.

1 Mein spezieller Dank gilt Silvia Capello, München, die mir im persönlichen Gespräch einiges Hintergrundwissen über die Forschung mit zerebralen Organoiden und die derzeit praktischen Limitierungen vermitteln konnte. Zudem danke ich Christoph Rehmann-Sutter, Lübeck, für hilfreiches Feedback zu einer früheren Fassung. 
Diese enorme Bedeutung, die dem Gehirn bei Wirbeltieren bei der Wahrnehmung, dem Schmerz- oder gar Selbstbewusstsein zukommt, hat andere dazu verleitet, gar über die Züchtung von Tieren ohne Köpfe nachzudenken, um dann z. B. derartig erzeugte Organe für die Forschung oder die regenerative Medizin zu verwenden. So erfuhr Mitte der 1990er Jahre der britische Forscher Jonathan Slack unerwartet viel Medienaufmerksamkeit, als er einem Journalisten seine entwicklungsbiologische Forschung mit dem Ziel der Züchtung von Fröschen „ohne Köpfe“ vorstellte (Die Zeit, 1999). Durch die fehlende neuronale, integrative Struktur des Gehirns könnten Slack zufolge moralische Probleme, die sich daraus ergeben, dass es sich bei den Forschungsansätzen um empfindungs- oder bewusstseinsfähige Wesen handeln könnte, biotechnisch gelöst werden. Inzwischen ist die Forschung an Organoiden um einiges weiter. Zugleich werden hier wieder ähnliche Fragen aufgeworfen, insbesondere wenn es sich um die Züchtung von sogenannten menschlichen „Mini-Hirnen“ handelt.

Diese zerebralen Organoide, d. h. gezüchtete Gewebegebilde, die neuronale Zellen beinhalten, spielen für die aktuelle neurobiologische Erforschung von psychiatrischen Erkrankungen wie Autismus, Depression, Schizophrenie oder Auswirkungen der Zika-Virus-Infektion auf die Gehirnentwicklung, aber auch für die Erprobung von medikamentösen Ansätzen eine sehr wichtige Rolle (siehe Tanaka/Park, Kap. 3.5). Hintergrund dessen ist auch, dass man davon ausgeht, dass einige dieser psychiatrischen Erkrankungen durch eine sogenannte Heterotopie, d. h. eine abweichende Organisation von Neuronen während der Hirnentwicklung, entstehen. Mit Organoiden kann man derartige abweichende Strukturorganisationen teilweise modellieren und entsprechend detailliert untersuchen (Rossi et al., 2018).

Bei zerebralen Organoiden handelt es sich um dreidimensionale, in vitro hergestellte Gebilde, die z. T. eine Differenzierung von verschiedenen Nervenzellen, wie sie in verschiedenen Bereichen des menschlichen Gehirns und auch im Großhirn vorkommen, aus- bzw. nachbilden. Sie sind bislang nicht strukturell identisch mit den Gewebestrukturen des natürlich gereiften, menschlichen Gehirns. Ihnen fehlen sowohl sensorische als auch motorische Bahnen, aber sie entwickeln durchaus verschiedene Zelltypen. Da menschliche Gehirne im Vergleich zu gängigen Tiermodellen in der Forschung (z. B. Maus oder Ratte) wesentlich differenziertere Nervenzellschichten im Gehirngewebe (d. h. Ausbildung und anatomische Anordnung verschiedener Nervenzelltypen) haben, wird in ihrer Nachbildung mittels humaner zerebraler Organoide ein wesentlicher Vorteil für die Forschung gegenüber der Verwendung von Tiermodellen gesehen. Invasive Forschung am lebenden menschlichen Hirn verbietet sich aus ethischer Sicht, da das Risiko von Schäden für Patient*innen viel zu groß ist. Die nächste Generation von Organoiden stellen sogenannte „Assembloide“ dar, bei 
denen Organoide unterschiedlicher Differenzierung zusammengelegt werden: Diese können sogar mit Organoiden, welche sensorischen Input oder motorischen Output modellieren, kombiniert werden (Paşca, 2019). Damit könnten größere, komplexere und ausgereiftere zerebrale Organoidmodelle entstehen (Chen et al., 2019). Es käme auch der Differenzierung des Gehirns in verschiedene Areale etwas näher.

Zerebrale Organoide werden vorrangig aus Fibroblasten von menschlichen Spender*innen gewonnen, aus denen dann in vitro humane induzierte pluripotente Stammzellen (hiPS-Zellen), dann Neuronen und schließlich dreidimensionale Zellgebilde gezüchtet werden. In manchen Ansätzen werden auch menschliche und tierische Zellen für die Züchtung von Organoiden gemischt. Organoide, welche nicht-zerebrale Organe wie z. B. die Leber nachbilden, werden auch aus humanen embryonalen Stammzellen (hES-Zellen) oder organspezifischen adulten Stammzellen gewonnen. Organoide aus Patientenzellen würden es gar ermöglichen, sehr patientenzentrierte, personalisierte Forschung zu betreiben. Gerade bei seltenen Erkrankungen oder Erkrankungen mit starker zellbiologischer Divergenz kann dies sehr hilfreich sein, aber für die breite Anwendung stellt dies sicher noch Zukunftsmusik dar.

Insgesamt speisen sich bei der Diskussion von zerebralen Organoiden viele Überlegungen zur ethischen Problemwahrnehmung aus bereits früher intensiv diskutierten Themen. Hierzu zählen insbesondere die menschliche Hirnforschung, die Spende und Verwendung von menschlichem Biomaterial sowie die Xenotransplantation (Übertragung von tierischen Zellen oder Organen auf den Menschen und umgekehrt). Es ist daher sehr wichtig, die Diskussion um zerebrale Organoide nicht losgelöst von diesen Zusammenhängen zu sehen. Vielmehr ist sie darin einzubetten, um langfristig ein konsistentes und widerspruchsfreies Bewertungsergebnis zu erzielen.

Organoide, insbesondere zerebrale Organoide, erhalten derzeit viel Aufmerksamkeit in der Forschung. Parallel dazu haben Neurowissenschaftler*innen, oft gemeinsam mit Ethiker*innen, auf eine Reihe ungelöster ethischer Fragen hingewiesen. Dieses Kapitel will im Folgenden vorrangig einen Überblick über diese internationale Diskussion geben. Mir scheint es jedoch verfrüht, schon mit sehr spezifischen Lösungsansätzen aufzuwarten, da die Debatte erst begonnen hat. Zudem ist es für eine fundierte ethische Bewertung von zentraler Bedeutung, erstmal überhaupt einen breiten, empirisch informierten Konsens darüber zu erzielen, was eigentlich die zentralen ethischen Probleme sind. Hierbei sollte zwischen forschungsethischen und medizinethischen Problemfeldern unterschieden werden (siehe Abschnitt 6.3).

Zuvor möchte ich aber darauf aufmerksam machen, dass einige ethische Fragen im Zusammenhang mit sowohl wissenschaftstheoretischen als auch grundlegenden neurophilosophischen Problemen stehen, die noch lange nicht eindeutig geklärt sind. 
Diese haben sowohl etwas mit den theoretisch-definitorischen Kategorien in der Beschreibung von Organoiden und ihren Eigenschaften zu tun (siehe zu konzeptuellen Fragestellungen auch Fagan, Kap. 4) als auch mit der Unterscheidung von fiktionalen Gedankenexperimenten und ethischer Folgenabschätzung (Abschnitt 6.2). Zudem muss die ethische Diskussion auch die kulturgeschichtliche und kulturelle Einbettung solcher Debatten berücksichtigen. Neben dem eingangs erwähnten Neurozentrismus ist hier auch die Diskussion um Mensch-Tier-Chimären und wie diese sowohl in der Wissenschaft, der Wissenschaftskommunikation als auch den Medien präsentiert werden bedeutend (Abschnitt 6.4). Daher komme ich zu dem Schluss (Abschnitt 6.5), dass uns das Thema Organoide noch eine Weile in der Wissenschafts- und Bioethik begleiten wird. Es bedarf sicher weiterer intensiver und insbesondere interdisziplinärer Auseinandersetzung und auch der Einbeziehung einer breiten Öffentlichkeit.

\subsection{Begriffliche Vielfalt auch als Ausdruck moralischer Verwirrung: Minihirne, Hirnorganoide, zerebrale Organoide oder Hirnmodelle?}

Während ein Großteil der Forschung noch damit kämpft, langlebige, funktionsdifferenzierte zerebrale Organoide aus menschlichen Zellen in vitro zu züchten, berichten einzelne Forscher*innen bereits von spektakulären Erfolgen. Für die einen ist ein Organoid, welches in Größe, Hirnreife und genetischer Komposition dem Gehirn eines fünf Wochen alten menschlichen Fetus entspricht, ein „Minihirn“ (Chapman, 2019) und manche sprechen gar von einem „Gehirn im Tank“ („brain in a vat“; Lavazza/Massimini, 2018). Andere Autor*innen betonen, dass die existenten Organoide „nur“ lokale Nervenzellstrukturen nachmodellieren, aber weder eine Hirnentwicklung über die Zeit leisten können (Sawai et al., 2019) noch ausreichende neuronale Vernetzung mit sensorischen und motorischen Neuronen haben. Daher ist es für Letztere auch höchst unwahrscheinlich, dass zerebrale Organoide in der nächsten Zeit mentale Eigenschaften entwickeln können. Allerdings sei dies nicht gänzlich auszuschließen, schreiten die Forschungstechniken weiter voran. Zumindest schließen diese Autor*innen aufgrund der raschen Erfolge der letzten Jahre in diesem Feld nicht aus, dass zerebrale Organoide künftig verschiedene Formen von kognitiven oder mentalen Fähigkeiten entwickeln können. Unter kognitiven Fähigkeiten verstehe ich hier die generelle Fähigkeit der Informationsverarbeitung. Mentale Fähigkeiten meinen hingegen geistige Fähigkeiten wie Wahrnehmung, zielgerichtetes Verhalten und Handlungsplanung. 
In diesem Sinne wird schon deutlich, dass die unterschiedlich verwendeten Bezeichnungen als „zerebrale Organoide“, „Mini-Hirne“ oder auch „Modelle“ nicht nur aus wissenschaftssoziologischer Sicht interessant sind, sondern durchaus unterschiedliche moralische Intuitionen evozieren bzw. abbilden. Wer von Hirnen spricht, wird eher ethische Bedenken bezüglich der Eigenschaften dieser Gebilde anmerken und gar Schutzansprüche formulieren. Wer hingegen von Modellen oder Zellgebilden redet, möchte den essenziellen Unterschied zwischen Original und Modell deutlich machen. Welcher Begriff nun angemessen ist, wird auch von den de facto nachgewiesenen oder zumindest plausiblen Eigenschaften von Organoiden abhängen. Es soll aber schon anzeigen, dass hier sprachliche Praxis von großer normativer Bedeutung ist.

Aus dem Spannungsfeld zwischen „noch nicht jetzt, aber irgendwann möglich“ resultiert meines Erachtens die besondere Brisanz der Diskussion um zerebrale Organoide. Es fällt in der aktuellen internationalen Diskussion auf, dass immer wieder sogenannte Gedankenexperimente bemüht werden, die da z. B. lauten: Was wäre, wenn ein „Hirnorganoid“ ähnlich einem menschlichen Gehirn im Tank existierte und zumindest teilweise Erinnerungen und mentale Eigenschaften der Zellspender*innen hätte? Was wäre, wenn ein Tier mit einem menschlichen zerebralen Organoid Eigenschaften wie menschliches Selbstbewusstsein entwickelte?

Während aktuelle rechtliche Regelungen und Forschungsethik-Kommissionen noch sehr gut ausblenden können, ob solche Gebilde mentale Eigenschaften ausbilden können, ist es Aufgabe einer zukunftsweisenden Bioethik, mögliche Entwicklungen oder zumindest derartige Bestrebungen frühzeitig zu antizipieren. Nur so können Vorschläge zur weiteren Weichenstellung innerhalb der Forschung Orientierung bieten. Allerdings ist bei dieser antizipierenden Gratwanderung genau zu unterscheiden, ob es sich um komplett fiktionale, empiriefreie Gedankenexperimente handelt oder um Hypothesen, die den Rahmen wissenschaftlicher Möglichkeiten nachvollziehbar ausloten. Aus meiner Sicht ist es für die angewandte bioethische Diskussion sinnvoll, auf Letzteres zu setzen. (Neuro-)Philosophische Gedankenexperimente haben im Rahmen der Überprüfung von Argumentationen einen heuristischen Wert und können für die kulturelle Wahrnehmung von Wissenschaft sehr aufschlussreich sein. Aber sie dürfen nicht dazu verleiten, praktische Urteile zu fällen. Es wäre ein Kategorienfehler, wenn empiriefreie Annahmen die wissenschaftliche oder soziale Praxis anleiteten.

\section{Exkurs 1: Gedankenexperimente vs. ethische Folgenabschätzung}

Gedankenexperimente sind fiktional. Sie können physikalische, biologische oder soziale Begebenheiten komplett ausblenden und sind in der Regel in der Philosophie 
so konzipiert, dass sie einen speziellen Unterschied in einer Argumentation veranschaulichen. Sie dienen der Überprüfung logischer Implikationen und begrifflicher Prämissen. Daher sind sie oft sehr reduktionistisch angelegt. Unter einer technischethischen Folgenabschätzung versteht man hingegen das Ausloten wahrscheinlicherer (oder auch eher plausibler) vs. unwahrscheinlicherer Zukunftsszenarien. Auch wenn darin also unter Unsicherheit oder Unwissenheit mögliche Entwicklungen antizipiert werden, müssen physikalische, biologische und soziale Kenntnisse unbedingt mitberücksichtigt werden. Diese unterzieht die Folgenabschätzung einer ethischen Bewertung; ob entsprechende Entwicklungen oder zumindest einzelne Folgen als erwünscht oder unerwünscht gelten oder gar moralisch klar abzulehnen sind. Bei solchen Folgenszenarien sind immer mehrere Faktoren der Veränderungen zu berücksichtigten, wodurch sie eine dichtere Beschreibung verschiedener Implikationen erlauben.

\subsection{Ein ethischer Überblick: „La lotta continua“}

Generell profitiert die bioethische Diskussion davon, wenn deutlich zwischen forschungsethischen und medizinethischen Überlegungen unterschieden wird. Bei medizinethischen Diskussionen geht es vorrangig um potenzielle Nutzen und Risiken für Patient*innen und darum, welche Rechte gegenüber einzelnen Patient*innen bzw. ganzen Gruppen zu wahren sind. Diese medizinethischen Fragen stellen sich dann, wenn Organoide als Organ- oder Gewebeersatz direkt zum klinischen Einsatz kommen. Da dieser breite Einsatz noch in der Zukunft liegt, soll er hier nur kurz behandelt werden. In der Forschungsethik geht es vorrangig darum, inwiefern die Forschung mit Organoiden menschlichen und tierischen Ursprungs eigene ethische Fragen aufwirft. Hierzu gehört auch, ob mit diesem Ansatz existente Forschungsansätze, die wiederum als ethisch problematisch gelten, gar ersetzt werden können.

\subsubsection{Medizinethische Aspekte}

Insbesondere bei nicht-zerebralen Organoiden wird diskutiert, wie diese in Form einer Organersatztherapie in Zukunft eingesetzt werden könnten. Lebergewebe, welches als hoch regenerativ gilt und keine komplizierte anatomische Struktur besitzt, bietet sich z. B. sehr gut an. So könnte vielleicht bald mittels humaner Leberorganoide chronisches Leberversagen therapiert werden (Bredenoord et al., 2017). Angesichts des andauernden Mangels an Organen für die Transplantation könnte sich somit eine wichtige medizinische Option ergeben. Natürlich müssen hier - vergleichbar zu allen anderen Verfahren - klinische Versuchsreihen gemäß den üblichen ethischen Stan- 
dards durchgeführt werden, um Nutzen und Risiken abzuschätzen. „Wild-West“-Manieren, wie sie z. B. bei einer ganzen Reihe von Stammzellanwendungen zutage treten, bei denen kommerziell geführte Kliniken nicht ausreichend geprüfte Stammzelltherapien an zahlende Patient*innen verabreichen, sind dabei unbedingt zu unterbinden (McLean et al., 2015; Sipp et al., 2017; Horner et al., 2018; siehe hierzu auch Besser et al., 2018).

Vielversprechend - im wahren Sinne des Wortes - sind Ansätze, welche im Kontext von personalisierter bzw. stratifizierter Medizin Biobanken mit patientenspezifischen Organoiden anlegen wollen, um etwa komplexe Erkrankungen $\mathrm{zu}$ erforschen und pharmakologische Behandlungen zu optimieren. Allerdings positionieren sich hier viele seriöse Forscher*innen eindeutig, dass man davon noch weit entfernt ist. Daher sollte aus medizinethischer Sicht, im Sinne des Respekts vor der Patientenautonomie, der Aufrichtigkeit gegenüber Patient*innen und der Öffentlichkeit sowie der Schadensbewahrung vor allem vom strategischen Gebrauch überzogener Heilsversprechen gewarnt werden. Es empfiehlt sich, dass Vertretende der Wissenschaft sowohl in der fachinternen als auch in der öffentlichen Kommunikation kritisch-reflexiv die Limitierungen dieser Forschung deutlich machen. Es ist aus ethischer Sicht zu verurteilen, wenn versucht wird, mit überzogenen Hoffnungen auf (zukünftige bzw. „mögliche“) Anwendungen sowohl soziale Akzeptanz als auch Fördermittel für diese Ansätze zu erschleichen. Andere Bereiche der biomedizinischen Forschung sind dafür bereits kritisiert worden (Murdoch/Scott, 2010; Brown, 2015).

\subsubsection{Forschungsethische Überlegungen}

Es stellen sich derzeit noch sehr viele Forschungsherausforderungen rund um die Züchtung funktionsfähiger und stabiler, $d$. h. langfristig kultivierbarer und funktionsfähiger, Organoide (siehe Kap. 3). Daran schließt sich eine Reihe forschungsethischer Probleme an. Einige dieser Probleme sind bereits sehr intensiv in Bezug auf die Stammzellforschung diskutiert worden (vgl. Bredenoord et al., 2017; Boers et al., 2019; Hyun et al., 2020). Forschungsethische Fragen betreffen sowohl nicht-zerebrale Organoide, wie solche für Augen, Darm oder Nieren, als auch zerebrale Organoide. Es lassen sich im Wesentlichen folgende vier Fragenkomplexe identifizieren:

\section{a) Können mittels Organoiden Tierversuche reduziert oder ersetzt werden?}

Eine große Hoffnung wird darauf gesetzt, dass die Forschung mit menschlichen Organoiden Tierversuche in der medizinischen Forschung reduzieren oder gar ersetzen 
kann. Die Reduktion bzw. der Ersatz von Tierversuchen werden sowohl ethisch als auch rechtlich in den letzten Jahren immer mehr gefordert. Da die bisher eher einfach gebauten Organoide in der Regel weder Blutgefäße noch Immunzellen besitzen und daher keine komplexere Organstruktur mimikrieren, bestehen jedoch noch Zweifel an der Möglichkeit, dass man dort auf Tierversuche verzichten kann, wo man bislang strikt für deren Unvermeidbarkeit argumentiert hat (z. B. weil nur sie die Untersuchung von komplexen physiologischen Zusammenhängen ermöglichen). ${ }^{2}$ In anderen Bereichen wie der Kosmetikentwicklung verzichten viele Firmen schon seit Längerem auf Tierversuche oder nutzen einfache toxikologische Tests mittels Gewebezüchtungen, da die Verbraucher*innen dies auch unterstützen und rechtliche Vorgaben entsprechend geändert wurden. Um den Ersatz von Tierversuchen in der biomedizinischen und pharmakologischen Forschung voranzutreiben, wären daher nicht nur umfangreiche Validierungsstudien sinnvoll, sondern auch, dass rechtliche Vorgaben zur Produktsicherheit geändert werden, da diese vielfach Tierversuche beinhalten.

Zudem ist bedenkenswert, dass trotz der Erfolge bei der Entwicklung von Ersatzund Reduktionsmethoden die Tierversuchszahlen in der medizinischen Forschung in den letzten Jahren nicht wirklich zurückgegangen sind. ${ }^{3}$ Daher steht und fällt diese ethisch begrüßenswerte Überlegung auch mit der wissenschaftlichen Weiterentwicklung von Organoiden in immer komplexere Gebilde, denn nur dann würde damit der Ersatz von Tierversuchen erfolgsversprechend sein (ebenso skeptisch: Bredenoord et al., 2017). Zudem haben Autor*innen darauf verwiesen, dass die Erforschung der Funktionsfähigkeit von etablierten Organoiden sogar weitere Tierversuche notwendig macht, um mögliche Risiken für spätere Patient*innen auszuschließen (Hyun et al., 2020). Insbesondere bei der Erzeugung von Mensch-Tier-Chimären mittels Organoiden muss die Auswirkung auf das Tierwohl kritisch mitbedacht werden. Engmaschige Studienprotokolle, die ebenfalls auch Tierschutzaspekte und ein Monitoring der Auswirkungen für die Versuchstiere im Blick haben, sind daher eine ethisch sinnvolle Forderung (Hyun et al., 2020).

2 Siehe dagegen für eine optimistische Einschätzung des Potenzials von Organoiden, Tierversuche langfristig zu ersetzen, das Interview mit Hans Clevers (Kap. 2.2).

3 Die Statistiken verzeichnen sogar einen enormen Anstieg, wenn die Anzahl gentechnisch erzeugter Tiere in diesen Statistiken mitberücksichtigt wird. Selbst wenn man diese jedoch rausnimmt, sind die Tierversuchszahlen in den letzten Jahren in Deutschland und anderen europäischen Ländern nicht nennenswert gesunken. Siehe unter: https://journals.sagepub.com/doi/abs/10.1177/02611929140420 0407 [30.06.2020]; http://kops.uni-konstanz.de/handle/123456789/32178 [30.06.2020]. 
b) Welche ethischen Bedingungen sind mit der Einwilligung in Biomaterialspenden und deren weiterer Verwendung einzuhalten?4

Sowohl für die Forschung als auch für die langfristige Verwendung von Zellen für die Organoidforschung ist die Etablierung von Biobanken absolut notwendig. Auch hier stellen sich gewissermaßen altbekannte ethische Fragen. Bereits in der Diskussion um die Etablierung von Biobanken anderer Zellen oder Gewebe kam die Frage nach einer ethisch akzeptablen Form der Einwilligung und ihrer Dauer auf. Insbesondere die ethisch begrüßenswerte Möglichkeit eines Widerspruchs, wie es dynamische Einwilligungsformen („dynamic consent“) erlauben, muss genau klären, ob existente Organoide wieder zerstört werden müssen, wenn Patient*innen später ihre Einwilligung zurückziehen. ${ }^{5}$ Weiterhin stellt sich die Frage, ob die Züchtung von Organoiden vorab geklärt wurde oder ob im Zuge des neuerdings von vielen favorisierten „broad consent" (d. h. der sehr generellen Zustimmung zur Verwendung von Daten oder Material für zukünftige Forschung, ohne die jeweiligen konkreten Forschungsziele oder -methoden zu kennen) auch Organoide gezüchtet werden dürfen - und wenn, dann welche und welche möchte man ausschließen? Es ist sehr wahrscheinlich, dass hier die persönlichen Ansichten von Patient*innen als Zellspender*innen stark divergieren.

Sinnvoll erscheint der Vorschlag, dass dort, wo die Forschung an Mensch-Tier-Chimären mit Organoiden möglich ist bzw. anvisiert wird, Spender*innen zumindest die Möglichkeit haben, solche Nutzungen auszuschließen (Farahany et al., 2018). Angesichts des noch recht neuen Ansatzes der Organoiderzeugung ist unklar, welche soziale und moralische Bedeutung Organoide für Spender*innen bzw. Patient*innen haben werden. Sollte es sich bei Organoiden um Gebilde handeln, zu denen Patient*innen evtl. eine moralisch relevante Beziehung aufbauen, gerade weil diese mehr als nur einfache Gewebe wären, dann scheint es nur konsistent, sie vorher um Einwilligung zu bitten. Daher könnte man auch von einem möglichen moralischen Wert von Organoiden sprechen, der zwar nicht dem absoluten Schutzanspruch entspricht, aber die Verwendung für (bestimmte) Forschungsvorhaben einschränken könnte (Boers et al., 2019). Dies könnte auch die Frage nach Eigentumsrechten (engl. „ownership“) bzw. den Rechten im Falle einer möglichen zukünftigen Kommerzialisierung oder Patentierung aufwerfen. Dies wäre insbesondere dann der Fall, wenn Organoide vom reinen Objekt-Status (also als „Ding“) zum Subjekt-Identitäts-Status (ein lebendiges „Wesen“) wechseln.

4 Für eine datenschutzrechtliche Einordung siehe Molnár-Gábor, Kap. 8.

5 Siehe auch Molnár-Gábor (Kap. 8) für eine ausführliche Diskussion der datenschutzrechtlichen Implikationen der Organoidforschung und verschiedener Formen der Einwilligung. 
c) Welcher moralische Status kommt den Zellen für die Erzeugung von Organoiden $z u$ ?

Adulte Stammzellen oder hiPS-Zellen gelten weithin als moralisch unbedenklich. Allerdings dort, wo Organoide nicht aus adulten oder hiPS-Zellen gewonnen werden, sondern aus hES-Zellen oder fetalem Gewebe, muss direkt auf die langanhaltende Kontroverse zur Gewinnung und Verwendung menschlicher Embryonen für die Forschung verwiesen werden. Die Erzeugung von hES-Zellen hat als eigenes ethisches Feld sehr viel Aufmerksamkeit erhalten. Aus meiner Sicht hat es zu einer polarisierenden Positionierung im deutschen Diskurs und international zu divergenten Regelungen geführt (Badura-Lotter, 2005; Joerden et al., 2009). Diesen Aspekt selbst will ich daher hier nicht weiterverfolgen und verweise auf die sehr gut aufgearbeitete Fachdiskussion. Befürworter*innen und Gegner*innen der humanen Embryonen- bzw. hES-Forschung stehen sich gegenüber und die deutsche Einzelfallbewertungsregelung für die Forschung hat meines Erachtens das Problem nicht substanziell gelöst, sondern nur prozedural verschoben. Es empfiehlt sich daher aus meiner Sicht, in der Organoidforschung die Nutzung von nicht-embryonalen humanen Quellen bevorzugt zu beforschen, wo es möglich ist. Natürlich ist das Fortführen der Debatte um eine zeitgemäße Embryonenforschung weiterhin sinnvoll und die aktuelle Pattsituation für viele unbefriedigend. Aber es ist zugleich diskursethisch problematisch, strategisch die Potenziale der Organoidforschung so darzustellen, dass diese ohne eine umfangreiche Embryonenforschung nicht auszuschöpfen wären. Denn insgesamt muss bedacht werden, dass bei solchen pluralistisch-kontroversen Themen praktisch-technischen Lösungen der Vorzug zu geben wäre. In diesem Sinne kann man auch die erfolgreiche Entwicklung von hiPS-Zellen verstehen.

\section{d) Welcher moralische Status kommt zerebralen Organoiden zu?}

Auch in Ländern, wo die Forschung mit menschlichen Embryonen bis zum 14. Tage oder die Erzeugung von hES-Zellen erlaubt ist, kann die Diskussion um den moralischen Status neu entflammen. Es ist zu klären, ob Organoide, allen voran zerebrale Organoide, Eigenschaften entwickeln könnten, die dann analog zum moralischen Status des fortgeschrittenen Embryos herangezogen werden, diesem einen (eingeschränkten oder absoluten) Schutzanspruch zusprechen (Hostiuc et al., 2019). Es überzeugen weder die „spezies-zentrische“ Argumentation (es erscheint mir unplausibel, dass etwas absolut schützenwert sei, nur weil es vom Menschen abstammt, denn das beträfe dann auch andere Zellen etc.) noch das Potenzialitätsargument (alles sei absolut moralisch schützenswert, was das Potenzial hat, sich zum Menschen zu entwickeln - hingegen 
halte ich es derzeit für unplausibel, dass sich aus Organoiden ein Mensch entwickeln könnte). Bei zerebralen Organoiden sind allerdings vor allem sentientistische Argumente genauer zu prüfen, die darauf abheben, dass zerebrale Organoide möglicherweise kognitive oder mentale Eigenschaften wie Bewusstsein oder Aufmerksamkeit, Schmerzwahrnehmung oder Lernfähigkeit entwickeln könnten.

Derzeit betonen eigentlich alle Autor*innen, dass die bisher existenten zerebralen Organoide diese Kriterien nicht erfüllen. Allerdings sei es eben nicht auszuschließen, dass mittels der Zusammenführung mehrerer Organoide, die unterschiedliche neuronale Aktivitäten ausüben, entsprechende Eigenschaften ausgebildet werden könnten (Farahany et al., 2018; Lavazza/Massimini, 2018; Chen et al., 2019; Sawai et al., 2019; Bayne et al., 2020; Hyun et al., 2020). Lavazza und Massimini sprechen daher auch vom Gedankenexperiment, welches nun zum Laborexperiment werde, und stellen so einen direkten Übergang von der Fiktion zum Faktischen her (606). Neben den Möglichkeiten, Schmerz oder Stress zu empfinden oder weiterzuleiten, sei dann auch nicht ausgeschlossen, dass diese Gebilde mentale Fähigkeiten wie Lernen, Erinnern, Wahrnehmen oder gar so etwas wie Bewusstsein entwickeln könnten. Dabei wird schnell deutlich, dass unser Verständnis von Bewusstsein weiterhin sehr vage und unscharf ist. Während manche Autor*innen damit Schmerzempfindungsfähigkeit meinen, und unzweifelhaft darauf aufmerksam machen, dass dies nun keine dem Menschen allein vorbehaltene Eigenschaft ist, verwenden andere den Begriff „consciousness“ in einer solchen Weise, dass mentale Eigenschaften wie Erinnerung, Reflexion und Selbstwahrnehmung gemeint sind. Je nach Verständnis von Bewusstsein verschieben sich somit auch die moralischen Kriterien: Für die einen ist es schon problematisch, wenn Organoide ähnlich einfacher Wirbeltiere oder sogar Mollusken Schmerzempfinden hätten. Für andere ist es moralisch problematisch, wenn Organoide komplexere mentale Eigenschaften entwickeln könnten und damit so etwas wie persönliche Identitätsmerkmale ausbildeten.

Es fällt auf, dass die Diskussion um Bewusstseinsfähigkeit von (zukünftigen) zerebralen Organoiden darunter leidet, dass es bislang kein einheitliches, zwischen Neurowissenschaften und Philosophie geteiltes Verständnis von Bewusstsein gibt (vgl. Rieger/Schicktanz, 2003: siehe auch die entsprechende Kritik bei Hyun et al., 2020), auf welches dann andere Disziplinen wie die Rechtswissenschaften oder die angewandte Ethik leicht zurückgreifen könnten. Nur einzelne Autor*innen in der Diskussion um die Fähigkeiten von zerebralen Organoiden weisen in Grundzügen ihre jeweiligen neurophilosophischen Prämissen aus. Manche unterscheiden zwischen drei Formen wie Vorbewusstem, einfachen kognitiven Funktionen und Bewusstsein oder auch zwischen dem Selbstbewusstsein (dem bewussten Vorhandensein eines Selbstkonzepts, 
z. B. als „ich“), dem phänomenalen Bewusstsein (i. S. eines subjektiven Erfahrens qualitativer Inhalte [Qualia]) und dem Zugangsbewusstsein (i. S. eines Zugangs zu externen Informationen) (siehe Sawai et al., 2019: 443). Es wird schnell deutlich, dass die Zuschreibungen alles andere als eindeutig und konsensfähig sind. Gerade aus sentientistischer Sicht ist interessant, wie unterschiedlich Empfindungsfähigkeit in diesem Spektrum von Bewusstseinsformen eingeordnet wird. Bei Chen et al. (2019) wird Empfindungsfähigkeit unter Selbst-Wahrnehmung am oberen Spektrum von Gehirnleistungen angeordnet und damit als besondere, rein menschliche Fähigkeit attribuiert. Andere betonen hingegen, dass viele Tiere, einschließlich derer, die oft in Tierversuchen eingesetzt werden, derartige Bewusstseinsformen und damit sowohl Aufmerksamkeits- als auch Empfindungsfähigkeit besitzen. Da dies kein menschliches Spezifikum sei, mache es auch wenig Sinn, dieser Problematik nur bei der Verpflanzung von menschlichen zerebralen Organoiden in Tiere Aufmerksamkeit zuzuwenden, sondern sei allgemein zu reflektieren (Hyun et al., 2020; Sawai et al., 2019: 443). Wenn dem so wäre, dann könnte sich gar mit der möglichen Steigerung von kognitiven Eigenschaften durch die Transplantation von zerebralen Organoiden auch der moralische Status dieser Versuchstiere ändern. Chen et al. (2019) gehen daher auch soweit, über verschiedene Szenarien des Enhancements (im Sinne einer Verbesserung oder gar Optimierung von bisherigen bio-physiologischen Eigenschaften) mittels menschlicher zerebraler Organoide bei Tieren zu spekulieren. Ihr Vorschlag ist, alle Ansätze zur Verpflanzung von menschlichen zerebralen Organoiden in Tiere als Enhancement zu diskutieren; anstelle einer Problematisierung der "Vermenschlichung“ von Versuchstieren bei Mensch-Tier-Chimären. Zugleich brechen sie dabei eine Lanze für eine derartige Forschung mit zerebralen Organoiden, um, wie sie einfordern, „realistischere“ Einschätzungen dieser Chimären-Hirnorganoid-Technik zu erhalten. Damit vertreten sie eine Position, die ein typisches Paradox der Forschungsethik enthält: Ein Mehr an ethisch problematischer Forschung sei notwendig, um die ethischen Probleme einer Forschung besser abschätzen zu können.

Die Frage nach den ethischen Implikationen, sollten zerebrale Organoide oder zumindest noch komplexere Formen von Assembloiden Bewusstseinsformen, Schmerzempfindung oder andere subjektive, mentale Zustände entwickeln können, beschäftigte zudem eine internationale siebzehnköpfige Expertengruppe mit Mitgliedern aus den Rechtswissenschaften, der Molekularbiologie, den Naturwissenschaften und der Ethik (Farahany et al., 2018). Sie hat in der Zeitschrift Nature 2018 auf eine ganze Reihe ungelöster ethischer Probleme hingewiesen, vor allem für den Fall, dass zerebrale Organoide sich immer mehr in die Richtung großer, komplexer und neuronal integrativer Strukturen entwickeln. Der Fakt, dass diese Expertengruppe betont, dass man 
zwar von solchen mentalen Eigenschaften bei Organoiden noch weit entfernt sei, ihre Realisierung aber nicht ganz ausschließen könne, ist dabei in zweierlei Hinsicht beachtenswert. Zum einen fordert die Forschergruppe selbst nicht nur die Fachwelt auf, sich damit zu beschäftigen, sondern auch die Zivilgesellschaft, geldgebende Institutionen sowie Ethiker*innen seien unbedingt einzubinden. Skepsis äußert die Gruppe, ob die bisher häufig involvierten lokalen Forschungsethikkommissionen wirklich kompetent seien, dieses komplexe Feld der Forschung angemessen zu beurteilen. Ihr Aufruf ist daher als ein wichtiger Anstoß zur Debatte zu verstehen. Zum anderen wird deutlich, dass sich das Spektrum angrenzender Fragen enorm erweitert, wenn man die Szenarien um empfindungsfähige oder bewusstseinsfähige zerebrale Organoide ausweitet und weiterdenkt. Die Expertengruppe fragt zu Recht, wie man denn überhaupt mentale Fähigkeiten messen könne: Sind neurowissenschaftliche Messmethoden wie das EEG oder andere nicht-invasive Neuroimaging-Methoden, die man beim lebenden Menschen nutzt, um Bewusstseinskorrelate festzustellen, auch bei Tieren, Chimären oder gar Organoiden einsetzbar? Die bisherigen Methoden haben enorme Beschränkungen für diesen Zweck, sodass man eher Methoden entwickeln müsse, die denen ähnlich sind, die bei Patient*innen im Koma eingesetzt werden (Lavazza/Massimini, 2018). Aus der fehlenden Messbarkeit darf allerdings nicht per se ein Nicht-Vorhandensein geschlossen werden - das wäre ein gewichtiger Kategorienfehler.

Ein ganz anderes Problem stellt sich der oben erwähnten Expertengruppe zufolge, wenn zukünftige zerebrale Organoide im Falle eines diagnostizierten Hirntods therapeutisch eingesetzt werden könnten. Damit würde die weit verbreitete Annahme (und auch rechtlich verankerte Voraussetzung) eines irreversiblen Hirntodes und damit die Gleichsetzung des Letzteren mit dem Tod des Menschen ins Wanken geraten. Allerdings können gegenwärtig Gebilde, die nur einige Neuronen oder begrenzte Bereiche der Gehirnaktivität ersetzen, nicht die klinische Funktionalität eines menschlichen Gehirns wieder in Gang setzen. Es bleibt daher genauer zu prüfen, ob die Forschung sich in diese Richtung wirklich entwickeln kann oder hier nur ein fiktionales Potenzial entwickelt wird.

Schließlich hat ein von der Expertengruppe gemachter Vorschlag besonderes mediales Interesse hervorgerufen, nämlich, dass über die rechtliche Vormundschaft für entsprechende zerebrale Organoide mit menschlichen, mentalen Eigenschaften, diskutiert werden müsse (FAZ, 2018). Das Vormundschaftsmodell (engl. „guardianship“) geht davon aus, dass manche Parteien ihre eigenen, gewichtigen Interessen nicht ausreichend gut selbst vertreten können (z. B. Kinder, Personen mit Demenz), aber Dritte diese antizipieren und vertreten können, sodass gesellschaftlich und rechtlich deren Interessenschutz gewahrt wird. Ein Vormund würde dann in rechtlichen oder 
sozialen Entscheidungen die Interessen des Organoids vertreten. Obwohl verschiedene Philosoph*innen dieses Konzept bereits für Tiere als nicht-menschliche Wesen mit Bewusstseinseigenschaften vorgeschlagen haben (siehe z. B. Sunstein/Nussbaum, 2004; White, 2013), hat es sich meines Wissens bislang nicht durchgesetzt. Dies liegt wohl auch daran, dass in dem Moment, wo man ein Vormundschaftskonzept einführt, eigentlich die absolute Schutzwürdigkeit eines Wesens anerkannt wird und sich damit dessen Einsatz bzw. Verwendung in der Forschung analog zum Instrumentalisierungsverbot beim Menschen weitgehend verbieten würde. ${ }^{6}$

Es bleibt zu bedenken, dass die moralische Schutzwürdigkeit von Organoiden nicht allein von der Potenzialität der ursprünglichen Zellen (also z. B. wenn es sich um embryonale Zellen handeln würde) oder ihrer De-facto-Eigenschaften (wie z. B. dem Besitz von mentalen Eigenschaften) abhängen muss. Wie Bredenoord et al. (2017) argumentieren, kann der moralische Status auch je nach Organoid-Komplexitätsgrad von einer relationalen Bewertung der ursprünglichen Spender*innen abhängig sein. Dann handelt es sich um einen relationalen moralischen Status oder eine extrinsische Wertzuschreibung, die ebenfalls mit verschiedenen Schutzansprüchen einhergehen kann. Ein solches Wertzuschreibungsmodell ist dann durchaus bedenkenswert, wenn Persönlichkeitsmerkmale (ob genetischer oder pathologischer Art) mit dem Organoid seitens des/der Spender*in assoziiert werden, und so ein relationales Verhältnis zu den „eigenen“ Organoiden hergestellt wird (Boers et al., 2019). Ob es solche sozialen Prozesse der Wertzuschreibung überhaupt gibt, bedarf allerdings umfangreicher empirischer Forschung. Boers et al. (2019) schlagen daher vor, systematisch zu erheben, ob Zellspender*innen bzw. Patient*innen solche Bezüge zu ,ihren“ Organoiden herstellen und wenn ja, unter welchen Bedingungen.

\subsection{Welche ethischen Grenzen sind bei der Chimären- Bildung unter der Verwendung von zerebralen Organoiden zu bedenken?}

Die praktischen Möglichkeiten und ethischen Grenzen der Erzeugung von MenschTier-Chimären mit Bezug auf die Organoidforschung erfährt besondere Aufmerksamkeit, auch von einigen Lebenswissenschaftler*innen (Farahany et al., 2018). Es wurden z. B. zerebrale Organoide menschlichen Ursprungs in Ratten implantiert, wo sich Blut-

6 Hyun et al. (2020) verweisen in ihrem ethischen Review noch darauf, dass zahlreiche Fragen bezüglich des moralischen Status von evtl. bewusstseinsfähigen zerebralen Organoiden ebenfalls Parallelen in der Diskussion zur künstlichen Intelligenz bzw. zu künstlichem Bewusstsein aufwerfen. 
gefäße ausgebildet haben, die diese Gebilde besser und länger versorgen. Dadurch ist eine mehrmonatige Existenz der Organoide im Tier ermöglicht worden (Levine/Grabel, 2017; siehe auch Tanaka/Park, Kap. 3.5). Zugleich sind durch das Tier natürliche Restriktionen in Bezug auf Wachstum und Entwicklung des Organoids vorgegeben. Bei Ratten kann das implantierte Organoid nicht sehr groß werden. Aber was wäre, wenn man andere Tiere, wie z. B. nicht-menschliche Primaten oder größere Säugetiere, verwendete (Chapman, 2019)? Die oben genannte Expertengruppe um Farahany et al. (2018) lehnt eine grundsätzliche Bewertung von Chimärenbildung ab: Sie plädiert für eine Fall-zu-Fall-Entscheidung. Ob dies jedoch im Kontext globaler und kommerzieller Forschung wirklich unethische Grenzüberschreitungen verhindert, muss man kritisch hinterfragen. Viel plausibler wäre es, grundsätzliche Grenzen zu definieren, die auf keinen Fall überschritten werden dürfen. Dazu bedarf es aber eines breiten gesellschaftlichen Diskurses und vermutlich auch initiierter Beteiligungsformate, die die Komplexität des Themas vielen Menschen zugänglich macht. Mehrere nationale gesetzliche Vorgaben und ethische Richtlinien sind insbesondere bei der Bildung von Mensch-Tier-Chimären, z. B. mit Bezug auf die Embryonenforschung, restriktiv (siehe auch Taupitz, Kap. 7).

Des Weiteren ist zu bedenken, dass die ethische Diskussion um Mensch-Tier-Chimären eine längere Geschichte aufweist. Bereits bei der Diskussion um die Erforschung der Transplantation von lebendigen, tierischen Organen, wie Herzen oder Nieren, auf den Menschen (sogenannte Xenotransplantation) hat dieses generelle Phänomen der Arten-Grenzüberschreitung gerade in der öffentlichen Wahrnehmung für viele Bedenken gesorgt (Schicktanz, 2002; Schicktanz, 2019). Andererseits scheitert die Verpflanzung von tierischen Organen in Menschen vor allem an der praxisnahen, berechtigten Sorge einer Übertragung von Zoonosen (Infektionskrankheiten) und den unzureichenden Organfunktionen. Für einige Patient*innen wäre ein Tierorgan als Ersatz absolut akzeptabel, für andere aus kulturellen oder religiösen Gründen nicht. Dementsprechend fällt der Chimärismus in seiner sozialen und moralischen Akzeptanz sehr unterschiedlich aus. Überlegungen, tierische zerebrale oder nicht-zerebrale Organoide beim Menschen als Organersatz bei Organversagen einzusetzen, werden mit dieser Ambivalenz ebenfalls rechnen müssen.

Gerade daher wird es wichtig sein, konsistente Regulierungen zu schaffen, welche auf einen breiten, gesellschaftlichen Konsens aufbauen, die sowohl diese soziokulturellen und pluralistischen Bedingungen reflektieren als auch Lösungen finden, die zugleich für alle Betroffenen akzeptabel erscheinen. Ob diese Lösung in einem schlichten liberalistischen Ansatz, jede*r solle selber entscheiden, liegt, halte ich allerdings für wenig überzeugend. Vielmehr müssen die Alternativen ausreichend ausgelotet 
und langfristige Konsequenzen, sowohl materieller als auch immaterieller Art, die viele betreffen, bedacht und abgewogen werden. In diesem Sinne muss ein ethisch wünschenswerter, gesellschaftlicher Konsens weit mehr Überlegungen einbeziehen, als dies der liberalistische Minimalkompromiss leisten kann.

\section{Exkurs 2: Zur kulturellen Bedeutung des Chimären-Motivs in der Öffentlichkeit}

Wissenschaftler*innen müssen sich darüber im Klaren sein, dass kein Motiv die gesellschaftliche Kontroverse über biotechnologische Forschung so befeuert hat wie das Chimären-Motiv. In den Lebenswissenschaften meint der Begriff des Chimärismus die Vermischung von Zellen zweier ursprünglich genetisch unterschiedlicher Wesen in einem neuen Zellverband. Zugleich stellt er einen Schlüsselbegriff zum Verständnis der Ambivalenz solcher und ähnlicher Forschungsansätze in ihrer populären Wahrnehmung dar, also bei Patient*innen sowie Laien, in den Medien oder in der Kunst. Hier lassen sich Hoffnungen und Ängste bezüglich der Verwischung der Grenzen von Eigen und Fremd, von Mensch und Tier, besonders verdeutlichen. Das Motiv der MenschTier-Chimäre spielte bereits in der öffentlichen Diskussion zur Xenotransplantation um das Jahr 2000 eine zentrale Rolle. Diese Technik wurde damals als neue Alternative zur gängigen Transplantationsmedizin beworben, jedoch zeitnah von vielen nationalen und internationalen Gremien mit einem Moratorium belegt. Nach zwei Dekaden in der Versenkung erfährt sie nun wieder eine Neubelebung. Alternative Begriffe wie „Mensch-Tier-Verbindung“ sind ebenfalls problematisch, da sie Assoziationen zur emotionalen Beziehung von Menschen zu Tieren wecken.

Die Verwendung der Begriffe „Mensch-Tier-Chimäre“ und „Chimärismus“ ist dabei nicht nur von einem Beigeschmack des Widernatürlichen, sondern auch des Göttlichen begleitet, der sich allenfalls aus den mythologischen und kulturhistorischen Wurzeln verstehen lässt. Gerade die kulturellen Referenzen verdeutlichen, dass bei ihrer Verwendung nie nur die anatomische Vermischung oder ,äußerliche“ Verwandlung gemeint ist, sondern immer auch mögliche Änderungen wesentlicher Charaktereigenschaften bzw. des eigenen Selbstverständnisses erwogen werden. Dies beginnt bereits mit dem oft zitierten Roman Frankenstein von Mary Shelley von 1818. Im Roman ist Frankenstein ein junger, engagierter Arzt, der mit Forschungseifer aus den Erkenntnissen von Anatomie, Chemie und Elektrizität heimlich eine neue Kreatur erst zusammensetzt und dann zum Leben erweckt. Während die Kreatur zu Beginn des Romans als sensibles, wenngleich äußerlich abschreckendes Wesen charakterisiert wird, führt die soziale Ablehnung und mangelnde Verantwortungsübernahme von Frankenstein, führen also die sozialen Umstände dazu, dass die Kreatur zum gefürchteten 
Monster wird (Schicktanz, 2019). Koplin und Massie (2019) nutzen den Vergleich zur Frankenstein-Geschichte, um zu argumentieren, dass die moralische Ablehnung von Organoiden, weil sie äußerlich abartig, unnatürlich oder eben unbekannt erscheinen, falsch sein könne. Entweder man unterschätze den möglichen moralischen Status eines Wesens aufgrund des Ekels, den das Wesen bei anderen hervorrufe, oder derartige Reaktionen blockierten gar eine sinnvolle, gut begründete Umgangsform, selbst wenn das Wesen keinen eigenen moralischen Status innehabe, wie man ihn anderen Wesen zuspreche. In diesem Sinne schlagen sie vor, dass man Organoide als Entitäten mit Respekt behandeln solle, weil wir den Spender*innen dieser Zellen gegenüber Respekt zollen. Für Koplin und Massie folgt aus diesem Respekt, dass man Organoide nur für sehr gewichtige Zwecke nutzen sollte, selbst wenn sie keine Empfindungsfähigkeit haben. Damit bleibt natürlich immer noch zu klären, was für wen einen gewichtigen Zweck darstellt. Ist Grundlagenforschung im Bereich psychiatrischer Krankheiten ausreichend wichtig? Wäre die nachweisbare Reduktion von Tierversuchen gewichtig genug? Die eingeforderten Antworten ergeben sich nicht automatisch. Allerdings sind derartige Überlegungen dennoch hilfreich, weil sie einen dritten Weg eröffnen: Die moralische Anerkennung von Entitäten kann auch jenseits der Frage nach dem moralischen Status oder der Klärung des Vorhandenseins von Bewusstsein (und ggf. seiner verschiedenen Formen) stattfinden. Sie hinge dann, ähnlich wie das oben erwähnte Konzept des relationalen moralischen Werts von extrinsischen Wertzuschreibungen ab. Damit wären Organoide nicht gänzlich frei verfügbar, aber es bedarf natürlich einer gesellschaftlichen Verständigung darüber, was die jeweilige Wertzuschreibung auch praktisch bedeutet. Ist Grundlagenforschung generell schon ausreichend oder nur medizinisch-motivierte Grundlagenforschung? Ist Forschung für Produktsicherheit bei neuartigen Kosmetik- und Chemieprodukten ausreichend gewichtig oder nur mit wissenschaftlichem Nutzen? Wie können solche Wertdiskurse angemessen geführt werden? Solche Fragen müssen natürlich noch bearbeitet werden, da der bisherige Debattenstand eher am Anfang steht.

\subsection{Schlussbetrachtungen und Ausblick}

Die hier vorgelegte Übersicht der aktuellen internationalen Debatte zu Organoiden, insbesondere zerebralen Organoiden und Mensch-Tier-Chimären, wollte deutlich machen, dass diese erst am Anfang steht. Es gibt mehr Fragen als Antworten. Damit besteht ein echter Bedarf für eine weiterführende bioethische Diskussion um Organoide im Allgemeinen und um zerebrale Organoide im Besonderen. 
Für diese weitere Diskussion ist Folgendes jedoch empfehlenswert:

Erstens sollte diese Auseinandersetzung auf keinen Fall losgelöst von den bereits sehr umfangreich geführten Diskussionen zur Spende und zum Umgang mit menschlichem Biomaterial, zu den tierethischen Dimensionen von Tierversuchen und der Frage nach den Grenzen möglicher Mensch-Tier-Chimären weitergeführt werden. Auch eine überzogene Rhetorik des Heilversprechens und die Darstellung von Organoiden als „Alleskönner“ oder „Wundermittel“ sollte sich aus ethischen Gründen verbieten. Da aus meiner Sicht ein gesellschaftlicher Konsens bezüglich der Akzeptanz von Embryonenforschung und der Verwendung von hES-Zellen eher in weiter Ferne liegt, scheint es pragmatisch sinnvoll, solche Forschungsansätze vorzuziehen, die ausschließlich mit hiPS-Zellen des Menschen arbeiten. Das vielversprechende Potenzial von Organoiden für die Forschung durch eine Wiederbelebung der Kontroverse um den moralischen Status des Embryos zu gefährden, wäre aus dieser Perspektive eher bedauerlich. Vor allem, weil ich auch Zweifel habe, dass die möglichen Erfolge der Organoide die bisherigen Gegner der Embryonenforschung umstimmen werden. Dafür steckt die Organoidforschung selbst noch zu sehr in den Kinderschuhen.

Zweitens sollte man sich sowohl von neurowissenschaftlich-entwicklungsbiologischer als auch neurophilosophischer Seite um eine Schärfung und Differenzierung der Begriffe und Beschreibung möglicher mentaler oder kognitiver Eigenschaften bei zerebralen Organoiden und Assembloiden bemühen. Man darf skeptisch bleiben, dass aus dieser Diskussion heraus ein so komplexes Phänomen wie Bewusstsein eindeutig für alle Bereiche geklärt werden kann. Dennoch ist die Schärfung der jeweiligen Konzepte zentral, um konkrete Eigenschaften zu benennen, deren ethische Implikationen dann weiter untersucht werden können. Grobe Konzepte wie „Bewusstsein“ helfen hingegen wenig weiter, sondern verschleiern eigentlich nur den Umstand, dass hier generell viele Fragen offen sind.

Drittens wäre es wünschenswert, dass die Bewusstseinsfrage bei zerebralen Organoiden, wie sie nun von mehreren Autor*innen angesprochen wurde (Lavazza/Massimini, 2018; Shepherd, 2018; Lavazza, 2019; Bayne et al., 2020), nicht dazu führt, dass die vielen anderen forschungsethischen, ethisch-praktischen Fragen zu sehr in den Hintergrund geraten. Gerade auf dieser Ebene scheint mir die von der oben genannten Expertengruppe geäußerte Sorge sehr nachvollziehbar, dass bestehende Ethik-Governance-Institutionen, wie lokale Ethikkommissionen, etwas überfordert sein könnten, diese vielfältigen, verschiedenen Dimensionen im Detail umfangreich abzuschätzen. Hier ist vielmehr spezielle Expertise erforderlich, um derartige Forschung ethisch und rechtlich gut zu begleiten. 
Viertens sei darauf hingewiesen, dass einzelne Autor*innen eine nicht ganz unproblematische Diskussion zur Mensch-Tier-Debatte befördern, indem sie das Argument der Humanisierung von Tieren bei der Chimärenbildung unkritisch aufgreifen. Dabei wird behauptet, dass insbesondere in der öffentlichen Diskussion die Chimärenbildung abgelehnt würde, weil man dort um die äußerliche Gestalt von Chimären bange, also Tiere mit menschlichen Gesichtszügen vor Augen hätte. Dies sei (und ist) wissenschaftlich sehr unrealistisch. Jedoch verkürzt diese Darstellung in vielerlei Hinsicht die kulturell tiefsitzende und weitverzweigte Auseinandersetzung mit Chimären (Ette/Wirth, 2019). Des Weiteren möchte ich zu bedenken geben, dass es vielmehr die zunehmende Grenzüberschreitung (nicht die Humanisierung des Tieres) der Lebenswissenschaften ist, die für ein Unbehagen sorgen kann. Im westlichen Recht und der westlich-zentrierten Ethik werden entsprechende anthropologische Prämissen zur Grenzziehung, wie abgrenzende Kategorien von Mensch und Tier, tot und lebendig, meinem und deinem Körper, ständig wiederholt und damit eher verstärkt. Chimären mit menschlichen Organoiden verkörpern, ähnlich denen in der Xenotransplantation, hingegen einen von vielen neuen biotechnologischen Ansätzen, welche derartige zentrale und immer wieder sonst verteidigte Abgrenzungskategorien plötzlich unterlaufen. Das führt zu Recht zu Inkohärenzen und Widersprüchen (Rehmann-Sutter, 1996) oder zumindest zum expliziten Bedarf, entsprechende Grenzziehungen gesellschaftlich direkt und explizit zu verhandeln. Zudem sind hierbei auch große kulturelle Unterschiede in der Bewertung wahrscheinlich, die es ebenfalls zu berücksichtigen gilt (siehe auch die Empfehlung der Global Neuroethics Summit Delegates [2018] zu Hirnforschung).

Damit komme ich zum fünften, abschließenden Punkt. Hier soll auf die moralische Notwendigkeit eines breiten, gesellschaftlichen Diskurses hingewiesen werden, den es bislang zu diesem Thema nicht gibt. Damit ist im Sinne des Paradigmas der Involvierung nicht nur die Information der Öffentlichkeit mittels Wissenschaftskommunikation oder Medien gemeint. Vielmehr sollten die vielfältigen Ansätze der deliberativen Einbeziehung von verschiedenen Interessensvertretenden sowie Vertretenden der Öffentlichkeit, die sich inzwischen etabliert haben (Schicktanz, 2009; Hansen et al., 2018), ${ }^{7}$ dafür genutzt werden.

7 Siehe zum Verfahren der bundesweiten Stakeholderkonferenz auch unter: https://praediadem.de /projektbeschreibung/ [30.06.2020]. 


\subsection{Literaturverzeichnis}

Badura-Lotter, G. (2005): Forschung an embryonalen Stammzellen: Zwischen biomedizinischer Ambition und ethischer Reflexion. Campus, Frankfurt am Main.

Bayne, T. et al. (2020): Are there islands of awareness? In: Trends in Neurosciences 43(1): 6-16.

Besser, D. et al. (2018): Ungeprüfte Stammzelltherapieangebote. In: Zenke, M. et al. (Hrsg.): Stammzellforschung. Nomos, Baden-Baden: 139-152.

Boers, S. N. et al. (2019): Organoids as hybrids: ethical implications for the exchange of human tissues. In: Journal of Medical Ethics 45(2): 131-139.

Bredenoord, A. L. et al. (2017): Human tissues in a dish: the research and ethical implications of organoid technology. In: Science 355(6322): eaaf9414. DOI: 10.1126/science.aaf9414.

Brown, N. (2015): Metrics of hope: disciplining affect in oncology. In: Health 19(2): 119-136. DOI: $10.1177 / 1363459314555239$.

Chapman, A. R. (2019): Brain models in a dish: Ethical issues in developing brain organoids. In: AJOB Neuroscience 10(3): 113-115.

Chen, H. I. et al. (2019): Transplantation of human brain organoids: Revisiting the science and ethics of brain chimeras. In: Cell Stem Cell 25(4): 462-472.

Die Zeit (1999): Der nette Dr. Frankenstein. 29.07.1999. Unter: https://www.zeit.de/1999/31/Der_nett e_Dr_Frankenstein [17.06.2020].

Ette, O./Wirth, U. (Hrsg) (2019): Kulturwissenschaftliche Konzepte der Transplantation. De Gruyter, Berlin/Boston.

Farahany, N. A. et al. (2018): The ethics of experimenting with human brain tissue. In: Nature 556: 429- 432.

FAZ (2018) = Frankfurter Allgemeine Zeitung: Braucht dieses Hirn einen Vormund? 25.05.2018. Unter: https://www.faz.net/aktuell/wissen/medizin-ernaehrung/ethik-in-der-forschung-braucht-dieses -hirn-einen-vormund-15600821.html [17.06.2020].

Global Neuroethics Summit Delegates (2018): Neuroethics questions to guide ethical research in the international brain initiatives. In: Neuron 100(1): 19-36.

Hansen, S. L. et al. (2018): Stakeholder-Beteiligung in der klinischen Forschung: eine ethische Analyse. In: Ethik in der Medizin 30: 289-305.

Horner, C. et al. (2018): Can civil lawsuits stem the tide of direct-to-consumer marketing of unproven stem cell interventions. In: NPJ Regenerative Medicine 3(5). DOI: 10.1038/s41536-018-0043-6.

Hostiuc, S. et al. (2019): The moral status of cerebral organoids. In: Regenerative Therapy 10: 118-122. Hyun, I. et al. (2020): Ethical issues related to brain organoid research. In: Brain Research, Online-Publikation 01.04.2020. DOI: 10.1016/j.brainres.2020.146653.

Joerden, J. C. et al. (Hrsg.) (2009): Stammzellforschung in Europa: Religiöse, ethische und rechtliche Probleme. Peter Lang Verlag, Frankfurt am Main. 
Koplin, J./Massie, J. (2019): Lessons from Frankenstein 200 years on: brain organoids, chimaeras and other 'monsters'. In: Journal of Medical Ethics, Online-Publikation 30.01.2020. DOI: 10.1136/ medethics-2019-105839.

Lavazza, A. (2019): What (or sometimes who) are organoids? And whose are they? In: Journal of Medical Ethics 45(2): 144-145.

Lavazza, A./Massimini, M. (2018): Cerebral organoids: ethical issues and consciousness assessment. In: Journal of Medical Ethics 44(9): 606-610.

Levine, S./Grabel, L. (2017): The contribution of human/non-human animal chimeras to stem cell research. In: Stem Cell Research 24: 128-134.

McLean, A. et al. (2015): Untested, unproven, and unethical: the promotion and provision of autologous stem cell therapies in Australia. In: Stem Cell Research \& Therapy 6(1): 12.

Murdoch, C. E./Scott, C. T. (2010): Stem cell tourism and the power of hope. In: American Journal of Bioethics 10(5): 16-23.

Paşca, S. P. (2019): Assembling human brain organoids. In: Science 363(6423): 126-127. DOI: 10.1126/ science.aau 5729.

Rehmann-Sutter, C. (1996): Frankensteinian knowledge? In: The Monist 79(2): 264-279. DOI: 10.5840/ monist199679214.

Rieger, J. W./Schicktanz, S. (2003): Wenn Du denkst, dass ich denke, dass Du denkst. Anmerkungen zur interdisziplinären Auseinandersetzung über das Bewusstsein. In: Herrmann, C. et al. (Hrsg.): Bewusstsein: Philosophie, Neurowissenschaften, Ethik. UTB, Stuttgart: 22-52.

Rossi, G. et al. (2018): Progress and potential in organoid research. In: Nature Reviews Genetics 19(11): 671-687.

Sawai, T. et al. (2019): The ethics of cerebral organoid research: Being conscious of consciousness. In: Stem Cell Reports 13(3): 440-447.

Schicktanz, S. (2002): Organlieferant Tier? Medizin und Tierethische Probleme der Xenotransplantation. Campus, Frankfurt am Main/New York.

Schicktanz, S. (2009): Zum Stellenwert von Betroffenheit: Öffentlichkeit und Deliberation im empirical turn der Medizinethik. In: Ethik in der Medizin 21: 223-234.

Schicktanz, S. (2019): Anmerkungen zur Geschichte der Transplantationsmedizin und ihrer ethischen und kulturellen Relevanz. In: Ette, O./Wirth, U. (Hrsg.): Kulturwissenschaftliche Konzepte der Transplantationsmedizin. De Gruyter, Berlin: 123-146.

Shelley, M. (1818/2013): Frankenstein und der moderne Prometheus. dtv, München.

Shepherd, J. (2018): Ethical (and epistemological) issues regarding consciousness in cerebral organoids. In: Journal of Medical Ethics 44(9): 611-612.

Shoemaker, S. (1963): Self-knowledge and self-identity. Cornell University Press, Ithaca.

Sipp, D. et al. (2017): Marketing of unproven stem cell-based interventions: A call to action. In: Science Translational Medicine 9(397): eaag0426. DOI: 10.1126/scitranslmed.aag0426.

Sunstein, C. R./Nussbaum, M. C. (Hrsg.) (2004): Animal rights: current debates and new directions. Oxford University Press, New York. 
White, S. (2013): Exploring different philosophical approaches to animal protection. In: Sankoff, P. et al. (Hrsg.): Animal law in Australasia: Continuing the dialog. Federation Press, Alexandria. 